\title{
Lower health-related quality of life predicts all-cause hospitalization among HIV- infected individuals
}

Leonard Emuren ${ }^{1,2,3^{*}}$ (D), Seth Welles ${ }^{1}$, Marcia Polansky ${ }^{1}$, Alison A. Evans ${ }^{1}$, Grace Macalino ${ }^{4,5}$, Brian K. Agan ${ }^{4, *^{*}}$ and Infectious Disease Clinical Research Program HIV Working Group ${ }^{4}$

\begin{abstract}
Background: Health-related quality of life (HRQOL) is a patient-centered outcome measure used in assessing the individual's overall functional health status but studies looking at $\mathrm{HRQOL}$ as a predictive tool are few. This work examines whether summary scores of HRQOL are predictive of all-cause hospitalization in the US Military HIV Natural History Study (NHS) cohort.
\end{abstract}

Methods: The Short Form 36 (SF-36) was administered between 2006 and 2010 to 1711 NHS cohort members whose hospitalization records we had also obtained. Physical component summary scores (PCSS) and mental component summary scores (MCSS) were computed based on standard algorithms. Terciles of PCSS and MCSS were generated with the upper terciles (higher HRQOL) as referent groups. Proportional hazards multivariate regression models were used to estimate the hazard of hospitalization for PCSS and MCSS separately (models 1 and 2 , respectively) and combined (model 3).

Results: The hazard ratios (HR) of hospitalization were respectively 2.12 times (95\% Cl: 1.59-2.84) and 1.59 times (95\% Cl: 1.19-2.14) higher for the lower and middle terciles compared to the upper PCSS tercile. The HR of hospitalization was 1.33 times (95\% Cl: 1.02-1.73) higher for the lower compared to the upper MCSS tercile. Other predictors of hospitalization were CD4 count $<200$ cells $/ \mathrm{mm}^{3}$ (HR $=2.84,95 \%$ Cl: 1.96, 4.12), CD4 count 200-349 cells $/ \mathrm{mm}^{3}$ ( $\left.\mathrm{HR}=1.67,95 \% \mathrm{Cl}: 1.24,2.26\right), \mathrm{CD} 4$ count $350-499$ cells $/ \mathrm{mm}^{3}$ ( $\left.\mathrm{HR}=1.41,95 \% \mathrm{Cl}: 1.09,1.83\right)$, plasma viral load $>50$ copies $/ \mathrm{mL}(H R=1.82,95 \% \mathrm{Cl}: 1.46,2.26)$, and yearly increment in duration of HIV infection $(H R=0.94,95 \%$ Cl: 0.93, 0.96) (model 3).

Conclusion: After controlling for factors associated with hospitalization among those with HIV, both PCSS and MCSS were predictive of all-cause hospitalization in the NHS cohort. HRQOL assessment using the SF-36 may be useful in stratifying hospitalization risk among HIV-infected populations.

Keywords: HIV, Human immunodeficiency virus, HRQOL, Health-related quality of life, HAART, Highly active antiretroviral therapy, PCSS, Physical component summary scores, MCSS, Mental component summary scores, Hospitalization

\footnotetext{
* Correspondence: leoemuren@yahoo.com; idcrp@idcrp.org

${ }^{1}$ Department of Epidemiology and Biostatistics, Dornsife School of Public Health, Drexel University, Philadelphia, PA, USA

${ }^{4}$ Infectious Disease Clinical Research Program, Department of Preventive

Medicine and Biostatistics, Uniformed Services University of the Health

Sciences, Bethesda, MD, USA

Full list of author information is available at the end of the article
}

(c) The Author(s). 2018 Open Access This article is distributed under the terms of the Creative Commons Attribution 4.0 International License (http://creativecommons.org/licenses/by/4.0/) which permits unrestricted use, distribution, and reproduction in any medium, provided you give appropriate credit to the original author(s) and the source, provide a link to the Creative Commons license, and indicate if changes were made. The Creative Commons Public Domain Dedication waiver (http://creativecommons.org/publicdomain/zero/1.0/) applies to the data made available in this article, unless otherwise stated. 


\section{Background}

Health-related quality of life (HRQOL) is primarily used as a patient-centered outcome measure to assess the individual's overall functional health status and for evaluating therapeutic interventions in chronic diseases including human immunodeficiency virus (HIV) infection and acquired immune deficiency syndrome (AIDS) [1, 2]. However, a few studies have also utilized HRQOL as a prognostic tool for predicting survival in people living with HIV/AIDS (PLWHA) [3-6], showing that HRQOL is useful as a risk stratification tool in HIV-infected individuals both in clinical trials and observational studies.

While HIV remains incurable, successful treatment with highly active antiretroviral therapy (HAART) has resulted in prolonged survival among PLWHA [7-9] and with the steady incidence of HIV in the United States [9], the prevalence of the disease and, by extension, the burden of the disease on the healthcare system will continue to rise. To mitigate the increasing burden of the disease on the healthcare system and improve the quality of life of infected individuals, it is important that PLWHA are clinically stable and in optimal functional health, free from medical/mental comorbidities or opportunistic infections, and have minimal hospitalizations. Poor HRQOL measures have been associated with higher utilization of healthcare resources among patients with other chronic diseases [10-12]. Among HIV-infected individuals, HRQOL has been shown to be associated with hospitalization and emergency department utilization [5]. The rate of hospitalization in the U.S. Military HIV Natural History Study (NHS) cohort was previously reported to be as high as 137 per 1000 person years (PYs) [13]. Given this high rate of hospitalization, it is important to evaluate factors that may predict hospitalization such as HRQOL, in the hope that appropriate interventions directed at modifiable risk factors such as CD4 count and medical/mental comorbidities that impact HRQOL can be instituted with the ultimate goal of reducing the high hospitalization rate.

While HRQOL may be measured using various instruments, the Research and Development (RAND) Short Form 36 (SF-36) is one of the more commonly used instruments both in clinical trials and observational studies. Although the Medical Outcome Studies for HIV questionnaire has been used in previous studies to predict mortality $[4,6]$, this instrument is disease-specific and its measured health dimensions are slightly different from that of the SF-36. Furthermore, an instrument's ability in predicting mortality may not necessarily prove its usefulness in predicting other relevant clinical end-points such as hospitalization. To the best of our knowledge there has been only one study that has specifically looked at how HRQOL predicts hospitalization in HIV-infected individuals, but this study used a different instrument, the EuroQol [5], and adjusted for only CD4 count and HIV plasma viral load. In this research, we investigate the usefulness of the RAND SF-36 in predicting hospitalization in the NHS cohort. Because HRQOL reflects an individual's overall physical and mental functional health status, we hypothesize that participants with lower HRQOL are more likely to be hospitalized compared to participants with higher HRQOL over the period of follow-up.

\section{Methods \\ Study cohort}

The NHS is a prospective multicenter continuous enrollment observational cohort of HIV-infected active duty military personnel and other beneficiaries from the Army, Navy/Marines and Air Force enrolled since 1986 [14-17]. Participants are followed at six medical centers in the U.S. Demographic data are collected at baseline and updated while medical and medication histories and standard laboratory studies are collected biannually. Blood samples obtained from participants in this cohort from scheduled visits are stored in a repository. All NHS participants provided informed consent, and approval for this research was obtained from the institutional review board at each participating site.

\section{Study participants}

The SF-36 questionnaire was administered to NHS participants at every other study visit (approximately 12-month intervals) from April 2006 to September 2010. For these analyses, one SF-36 response per calendar year was captured, using the last measurement if more than one survey was completed in a calendar year. Baseline was defined as the earliest measure meeting these criteria. We used the CD4 count and viral load values closest in time to the HRQOL measure, usually the same visit.

\section{Definitions and variable selections Hospitalization}

Participants' dates of hospitalization, diagnoses at hospitalization, and number of days of hospitalization were retrieved from their medical records and through coordinator interviews. Furthermore, the military healthcare system operates a centralized electronic health records system that enables investigators to track participants' records and hospitalizations. Hospitalization was the outcome of interest, and we considered only the first admission of participants between April 2006 and September 2010 for the purposes of this study. To establish a temporal relationship, we ensured that date of completed questionnaire preceded the date of hospitalization. Hospitalization was coded as 'yes' if participant was hospitalized after the first completed SF-36 questionnaire and 'no' if participant was not hospitalized after the first completed questionnaire for the duration of 
follow-up. Some common causes of hospitalization include bacterial, viral, fungal and parasitic infections, cancers, psychiatric conditions such as major depressive disorders, alcohol abuse, gastroenterological disorders such as gastroesophageal reflux disease and peptic ulcers, and cardiovascular conditions such as myocardial infarction and pericarditis.

\section{Health-related quality of life scores}

The norm-based physical component summary scores (PCSS) and mental component summary scores (MCSS) were computed according to the recommended scoring algorithm for the RAND 36-item health survey $1.0[18,19]$. PCSS and MCSS, the main explanatory variables, were categorized into terciles with the upper terciles (highest HRQOL) as referent groups. Terciles were established separately for PCSS and MCSS using all available HRQOL scores and we verified an approximately even distribution of the number of participants in each tercile at baseline. Participants could move from one tercile to the other during the period of follow up based on their scores.

\section{Covariates}

Highly active antiretroviral therapy (HAART) was defined as a combination of at least three full dose antiretroviral agents similar to previous investigations for this cohort [15]. In light of prior reports that use of protease inhibitors (PI) is associated with poorer HRQOL [20-22], HAART was divided into four groups: protease inhibitor-based HAART (PI-HAART), for HAART with at least one protease inhibitor in the HAART regimen; non-protease-inhibitor-based HAART (NPI-HAART), for HAART regimens with no protease inhibitor; HAART-naïve group (HAART-N) for those who had never been on HAART, and Off-HAART/Non-HAART antiretroviral (ART) group, made up of those who were previously treated, but off HAART or who were on non-HAART ART. Other covariates were gender (male/ female), age (in increments of 5 years), military rank (officer/warrant officer, enlisted, and civilian/retired), marital status (married, not married), race/ethnicity (Caucasian, African-American, and others), HIV plasma viral load ([pVL], $\leq 50$ copies $/ \mathrm{mL},>50$ copies $/ \mathrm{mL}), \mathrm{CD} 4$ count $(<$ 200 cells $/ \mathrm{mm}^{3}, 200-349$ cells $/ \mathrm{mm}^{3}, 350-499$ cells $/ \mathrm{mm}^{3}$ and $>499$ cells $/ \mathrm{mm}^{3}$ ), medical comorbidity (yes/no), mental comorbidity (yes/no), AIDS diagnosis (yes/no), and time since HIV diagnosis (in years). AIDS was defined in accordance with the 1993 Centers for Disease Control and Prevention revised criteria, except for an isolated CD4 cell count $<200$ cells $/ \mathrm{mL}$, as CD4 was analyzed separately. Medical co-morbidity referred to chronic medical conditions such as diabetes mellitus, hypertension or cancer and was classified as having no comorbidity or having one or more comorbidity. Mental comorbidity was classified similarly. Common mental comorbidities in the NHS were major depressive disorder, generalized anxiety disorder, bipolar disorder and alcohol abuse. Except for gender and race, all other variables were treated as time-varying covariates. Baseline was earliest SF-36 captured according to the above criteria; follow-up continued to hospitalization, loss to follow-up, or Sept 30, 2010, whichever came first. Participants were deemed lost to follow up for this HRQOL analysis if they did not have a completed SF-36 questionnaire for a given year and none thereafter. Participants lost to follow-up were censored at 6 months after the last completed SF-36 or at Sept 30, 2010. All participants aged 18 years and above who were enrolled into the HRQOL sub-study between 2006 and 2010 were eligible for this analysis.

\section{Statistical analyses}

We summarized the characteristics of the participants based on their frequency distribution for categorical variables and the median and interquartile range (IQR) for continuous variables. We plotted the Kaplan-Meier curves to estimate the survivor functions by group using the log-rank test; the Tukey-Kramer adjustment was used to assess between-group differences for variables with more than two categories. Cox regression modeling was used to estimate the hazard of hospitalization for participants while adjusting for covariates. As above, all covariates were time varying, with the exceptions of gender and race. Because separate multivariate models are traditionally used for PCSS and MCSS when these variables are the outcome variables in research settings, we also used them separately as independent variables in two different models while controlling for the same set of covariates (models 1 and 2, respectively). Furthermore, we constructed a third model with both PCSS and MCSS included (model 3). To be eligible for inclusion into the multivariate models, the covariate must achieve a significance level of $<0.2$ in the univariate model. Missing data were handled using the last-observation-carried-forward method. In line with the model specifications, we verified the proportional hazard assumptions using both graphical and formal diagnostic tests including covariate-time interaction effects [23-25]. All statistical analyses and graphs were performed using SAS 9.3 [SAS Institute Inc., Cary, NC].

\section{Results}

Out of the 1730 eligible participants at baseline, 13 did not completely answer the HRQOL questionnaire and were excluded. Another 6 participants with missing values for one or more covariates at baseline were also excluded. Of the remaining 1711 participants included in this analysis, 366 (21\%) were hospitalized at least once 
Table 1 Demographic and Clinical Characteristics of Participants at Baseline

\begin{tabular}{|c|c|}
\hline \multicolumn{2}{|l|}{ Categorical Variables } \\
\hline Characteristics & $N(\%)$ \\
\hline \multicolumn{2}{|l|}{ Hospitalized } \\
\hline Yes & 366 (21.39) \\
\hline No & $1345(78.61)$ \\
\hline \multicolumn{2}{|l|}{ Gender } \\
\hline Male & $1594(93.16)$ \\
\hline Female & $117(6.84)$ \\
\hline \multicolumn{2}{|l|}{ Race } \\
\hline Non-Hispanic White & $719(42.02)$ \\
\hline Non-Hispanic African & $722(42.20)$ \\
\hline Others & $270(15.78)$ \\
\hline \multicolumn{2}{|l|}{ Rank } \\
\hline Officer/Warrant Officer & $126(7.36)$ \\
\hline Enlisted & $893(52.19)$ \\
\hline Others (Retired/Civilians) & $692(40.44)$ \\
\hline Marriage, Yes & $556(32.50)$ \\
\hline Medical Comorbidity, Yes & $285(16.66)$ \\
\hline Mental Comorbidity, Yes & $493(28.81)$ \\
\hline AIDS Diagnosis, Yes & $202(11.81)$ \\
\hline \multicolumn{2}{|l|}{ HAART } \\
\hline Pl-Based & $575(33.61)$ \\
\hline Non-PI-Based & $756(44.18)$ \\
\hline HAART-Naïve & $241(14.09)$ \\
\hline Off-HAART & $121(7.07)$ \\
\hline Non-HAART ART & $18(1.05)$ \\
\hline \multicolumn{2}{|l|}{ Plasma Viral Load > 50 copies/mL } \\
\hline Yes & $600(35.07)$ \\
\hline No & $1111(64.93)$ \\
\hline \multicolumn{2}{|l|}{ CD4 Count } \\
\hline$<200$ cells/mm3 & $92(5.38)$ \\
\hline 200-349 cells/mm3 & $242(14.14)$ \\
\hline 350-499 cells/mm3 & $411(24.02)$ \\
\hline > 499 cells/mm3 & $966(56.46)$ \\
\hline \multicolumn{2}{|l|}{ Continuous Variables } \\
\hline Characteristics & Median (Interquartile Range) \\
\hline Age (years) & $42.00(34.00-49.00)$ \\
\hline CD4 Count (cells/mm3) & $538.00(389.00-721.00)$ \\
\hline Plasma Viral Load (Log10) & $1.70(1.68-2.82)$ \\
\hline Time Since HIV Diagnosis (years) & $10.00(4.00-17.00)$ \\
\hline Duration of Follow-Up from Baseline (Years, Overall) & $2.71(1.04-3.80)$ \\
\hline Hospitalized & $1.23(0.53-2.37)$ \\
\hline Not Hospitalized & $3.13(1.53-3.95)$ \\
\hline \multicolumn{2}{|l|}{ Physical Component Summary Scores (PCSS) } \\
\hline Lower Tercile & $41.78(35.88-46.13)$ \\
\hline Middle Tercile & $54.56(52.78-55.87)$ \\
\hline Upper Tercile & $58.81(57.87-59.76)$ \\
\hline
\end{tabular}

Table 1 Demographic and Clinical Characteristics of Participants at Baseline (Continued)

\begin{tabular}{ll}
\hline Mental Component Summary Scores (MCSS) & \\
Lower Tercile & $39.18(31.99-43.88)$ \\
Middle Tercile & $50.69(49.02-51.82)$ \\
Upper Tercile & $55.22(54.04-57.29)$ \\
\hline
\end{tabular}

(Table 1). Participants were predominantly male (93\%), with equal representation from non-Hispanic Whites and African-Americans ( $42 \%$ each). $17 \%$ of participants had a medical comorbidity while $29 \%$ had a mental comorbidity, and about $12 \%$ had an AIDS diagnosis. Slightly over $5 \%$ of the cohort had CD4 count $<200$ cells $/ \mathrm{mm}^{3}$ and over $56 \%$ had CD4 count $>499$ cells/ $\mathrm{mm}^{3}$. $35 \%$ of participants had $\mathrm{pVL}>50$ copies $/ \mathrm{mL}$. About $34 \%$ of patients were on a PI-based HAART while $44 \%$ were on a non-PI-based HAART. $14 \%$ of participants were HAART naïve at baseline and another 7\% were off HAART at baseline. Only $1 \%$ of participants $(n=18)$ were on a non-HAART antiretroviral therapy and were combined with the Off-HAART group for the subsequent analyses.

The terciles for the PCSS at baseline had 570, 572 and 569 participants respectively in the lower, middle and upper terciles. The median score of the lower PCSS tercile was 41.78 (IQR, 35.88-46.13) compared to 54.56 (IQR, 52.78-55.87) for the middle tercile and 58.81 (IQR, 57.87-59.76) for the upper tercile. The Kaplan-Meier product-limit survival estimates for hospitalizations among the PCSS terciles showed statistically significant differences among all terciles of PCSS (Fig. 1). In the unadjusted Cox regression model, the hazard ratio (HR) of hospitalization for participants in the lower PCSS tercile was 2.52 times higher compared to participants in the upper PCSS tercile (95\% confidence interval [CI] 1.92-3.32; Table 2), while the hazard of hospitalization was 1.74 times higher in the middle tercile compared to the upper tercile (95\% CI: 1.31-2.33). After adjustment, the hazards of hospitalization were 2.12 (95\% CI: $1.59-2.84$ ) and 1.59 (95\% CI: 1.19-2.14) times higher respectively in the lower and middle terciles compared to the upper tercile (Table 3, model 3 [after this, we report model 3, which is the combined PCSS and MCSS model, unless there are obvious differences in the results of the three models]).

The terciles for the MCSS at baseline had 569, 571, and 571 participants respectively for the lower, middle and upper terciles. The median score of the lower MCSS tercile was 39.18 (IQR, 31.99-43.88) compared to 50.69 (IQR, 49.02-51.82) for the middle tercile and 55.22 (IQR, 54.04-57.29) for the upper tercile. The 


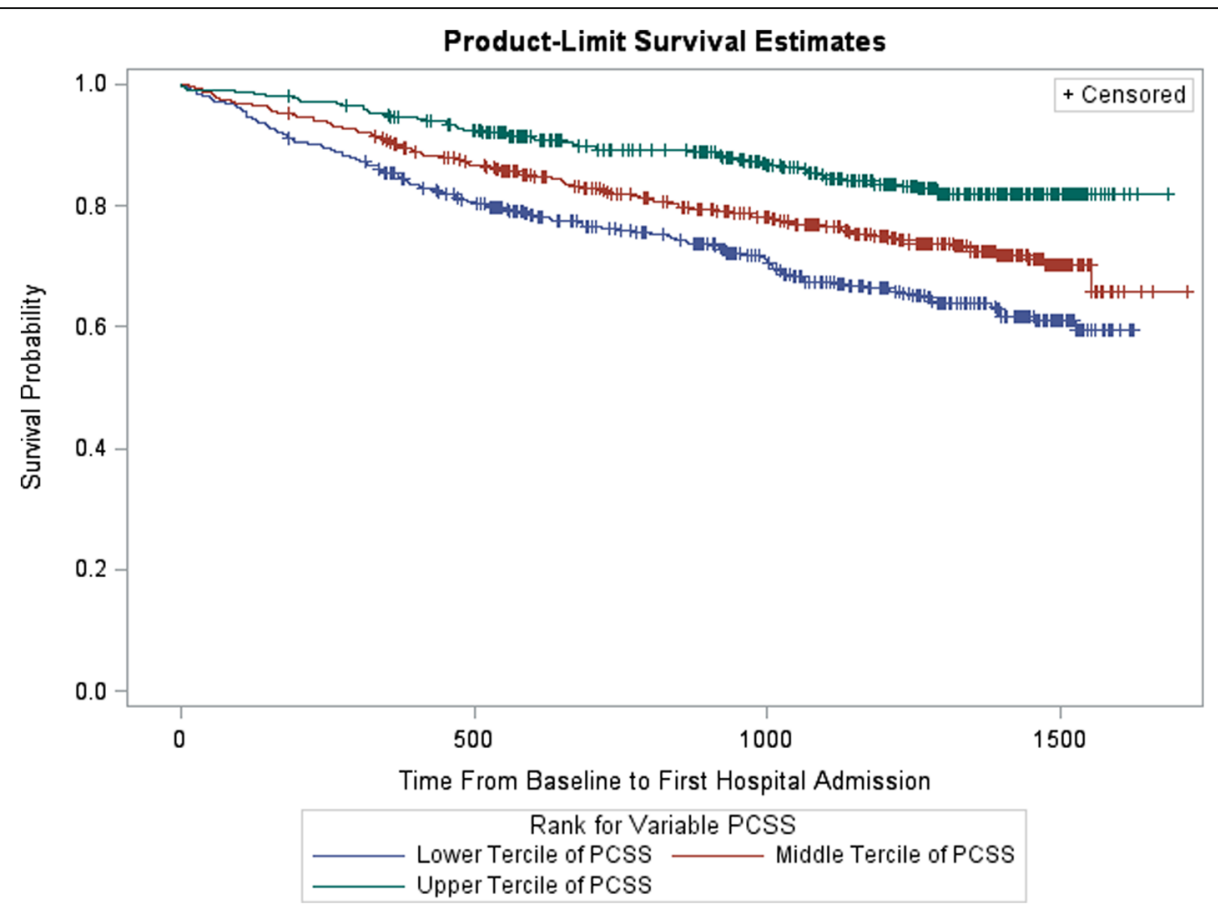

Fig. 1 Kaplan-Meier Survival Curve for Physical Component Summary Score (PCSS). Legend: Middle Tercile of PCSS,

Kaplan-Meier product-limit survival estimates for hospitalizations among the MCSS terciles showed statistically significant differences between the lower tercile and other two MCSS terciles, but not between the middle and upper terciles (Fig. 2). In the unadjusted Cox regression model (Table 2), participants in the lower MCSS tercile were $79 \%$ at increased hazard of being hospitalized compared to those in the upper MCSS tercile (HR: 1.79; 95\% CI: 1.39-2.30). After adjustment (model 3), the hazard of hospitalization in the lower tercile was $33 \%$ higher than that of the upper tercile (HR: 1.33; 95\% CI 1.02-1.73).

The first-hospitalization rate among the 1711 participants was 8.7 per 100 person-years (PYs). As previously noted, the number of participants at baseline in the lower, middle and upper PCSS terciles were 570, 572 and 569 and the total duration of follow up in years were respectively $1312.34,1414.88$ and 1471.44 . There were 168 hospitalizations in lower terciles for the period of follow up, 124 in the middle tercile and 74 in the upper tercile, therefore making the rates of hospitalization $12.8,8.8$ and 5.0 per 100 PYs for the lower, middle and upper terciles respectively (Table 4). Similarly, the rates of hospitalization were 11.8, 8.3 and 6.5 per 100 PYs for the lower, middle and upper terciles of MCSS respectively (Table 4).

The HRs of hospitalization were significantly increased in participants with CD4 count $<200$ cells $/ \mathrm{mm}^{3}, 200-$
$349 \mathrm{cell} / \mathrm{mm}^{3}$ and $350-499 \mathrm{cell} / \mathrm{mm}^{3}$ by 2.84 (95\% CI: 1.96-4.12), 1.67 (95\% CI: 1.24-2.26), and 1.41 (95\% CI: 1.09-1.83) times respectively when compared to those with CD4 count $>499$ cells $/ \mathrm{mm}^{3}$. Having pVL > 50 copies/mL was significantly associated with $82 \%$ increase in the hazard of hospitalization (HR: 1.82; 95\% CI: $1.46-$ 2.26) while being retired/civilian was significantly associated with over a $100 \%$ increase in hazard of hospitalization (HR: 2.04; 95\% CI: 1.25-3.34) (Table 3). Every year increment in time from HIV diagnosis led to a 5.6\% reduced hazard of hospitalization (HR: 0.94; 95\% CI: 0.93-0.96). Factors that were not significantly associated with hospitalization in the combined model but significant in the PCSS and/or MCSS models (Table 3) were mental comorbidity (both PCSS and MCSS models) and AIDS diagnosis (MCSS model only). HAART, on the other hand, was only significant in the univariate model but not in any of the multivariate models and therefore not shown in our most parsimonious models.

\section{Discussion}

Our study shows that both PCSS and MCSS were independently predictive of hospitalization among participants in the NHS cohort. To the best of our knowledge, this is the first study to use the SF-36 to evaluate whether HRQOL predicts hospitalization in an 
Table 2 Univariate Cox Regression Model for Hazard of Hospitalization

\begin{tabular}{|c|c|c|c|}
\hline Variable $^{a}$ & Hazard Ratio & $95 \% \mathrm{Cl}$ & $P$-Value \\
\hline \multicolumn{4}{|c|}{ Physical Component Summary Score (PCSS) } \\
\hline Lower Tercile of PCSS & 2.52 & $1.92-3.32$ & $<.0001$ \\
\hline Middle Tercile of PCSS & 1.74 & $1.31-2.33$ & 0.0002 \\
\hline Upper Tercile of PCSS & 1.0 & - & - \\
\hline \multicolumn{4}{|c|}{ Mental Component Summary Score (MCSS) } \\
\hline Lower Tercile of MCSS & 1.79 & $1.39-2.30$ & $<.0001$ \\
\hline Middle Tercile of MCSS & 1.27 & $0.97-1.67$ & 0.08 \\
\hline Upper Tercile of MCSS & 1.0 & - & - \\
\hline Age (Years, Increment of 5 Years) & 0.98 & $0.94-1.03$ & 0.5 \\
\hline Gender (Male) & 1.06 & $0.70-1.62$ & 0.8 \\
\hline Marital Status (Married) & 1.13 & $0.91-1.40$ & 0.3 \\
\hline \multicolumn{4}{|l|}{ Race/Ethnicity } \\
\hline Non-Hispanic African American & 0.96 & $0.77-1.19$ & 0.7 \\
\hline Hispanic/Others & 0.86 & $0.63-1.18$ & 0.3 \\
\hline Non-Hispanic Caucasian & 1.0 & - & - \\
\hline \multicolumn{4}{|l|}{ Rank } \\
\hline Civilian/Retired & 1.47 & $0.93-2.35$ & 0.1 \\
\hline Enlisted & 1.22 & $0.77-1.95$ & 0.4 \\
\hline Officers & 1.0 & - & - \\
\hline \multicolumn{4}{|l|}{ CD4 Count } \\
\hline CD4 Count $<200$ cells/mm3 & 3.89 & $2.78-5.44$ & $<.0001$ \\
\hline CD4 Count 200-349 cells/mm3 & 2.16 & $1.63-2.86$ & $<.0001$ \\
\hline CD4 Count 350-499 cells/mm3 & 1.55 & $1.20-2.00$ & 0.0008 \\
\hline CD4 Count > 499 cells $/ \mathrm{mm} 3$ & 1.0 & - & - \\
\hline Plasma Viral Load > 50 Copies $/ \mathrm{mL}$ & 2.36 & $1.92-2.89$ & $<.0001$ \\
\hline Medical Comorbidity & 1.05 & $0.81-1.36$ & 0.7 \\
\hline Mental Comorbidity & 1.43 & $1.16-1.76$ & 0.0009 \\
\hline AIDS Diagnosis & 1.67 & $1.27-2.18$ & 0.0002 \\
\hline Time Since HIV (Per year) & 0.98 & $0.96-0.99$ & 0.002 \\
\hline \multicolumn{4}{|l|}{ HAART Treatment } \\
\hline HAART-Naïve & 1.80 & $1.33-2.43$ & 0.0002 \\
\hline Off-HAART/Non-HAART ART & 1.75 & $1.26-2.44$ & 0.0008 \\
\hline Non-PI Based HAART & 0.70 & $0.55-0.87$ & 0.003 \\
\hline PI Based HAART & 1.0 & - & - \\
\hline
\end{tabular}

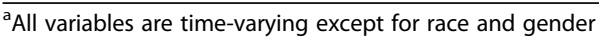

HIV-infected population, and perhaps the second study for any HRQOL instrument [5]. Our study therefore adds to the growing literature on the role of HRQOL in predicting hospitalization in chronic diseases [11, 2628]. In our current study, those in the middle PCSS tercile were at $59 \%$ greater hazard of hospitalization compared to the upper PCSS tercile while those in the lower PCSS tercile were at $112 \%$ greater hazard of hospitalization compared to the upper PCSS tercile. Participants in the lower MCSS tercile had a 33\% increased hazard of hospitalization compared to the upper MCSS tercile. These findings support the use of HRQOL in risk assessment for hospitalization in clinical and research settings. Although clinical factors such as mental comorbidity and AIDS diagnosis were not significantly associated with hospitalization in the combined model (model 3 , Table 3), mental comorbidity was significant in the separate PCSS and MCSS models while AIDS diagnosis was significant in the MCSS model and was nearly significant in the PCSS model. These covariates should therefore be included in studies examining clinical interventions directed at reducing the risk of hospitalization in PLWHA considering that they contribute to both the independent and outcome variables.

The overall rate of hospitalization of 8.7 per 100 PYs in our current study is much lower than that reported earlier for the NHS by Crum-Cianflone et al. [13], which reported a hospitalization rate of 137 per 1000 PYs or 13.7 per 100 PYs. This difference may be partly explained by the fact that we only considered the first hospitalization per individual as against all hospitalizations used in the study by Crum-Cianflone et al. Indeed, in that study $47 \%$ of all hospitalizations were repeat inpatient admissions. Furthermore, the general decline in hospitalization rates in HIV-infected populations over the years may partly explain the differences in hospitalization rates noted in both studies. The rates of hospitalizations were also markedly different by terciles of PCSS and MCSS. These rates were 5.0, 8.8 and 12.8 per 100 PYs for the upper, middle and lower PCSS terciles respectively, and 6.5, 8.3 and 11.7 per 100 PYs for the upper, middle and lower terciles of MCSS respectively. This clear pattern of less hospitalization with better physical and mental functional health status suggests that applying innovative research to delineate interventions that will improve HRQOL may be strategically significant in reducing hospitalization in our population and likely more broadly among PLWHA.

Our findings on the association among CD4 count and hospitalization were similar to and reinforced prior work. Compared to CD4 count $>499$ cells $/ \mathrm{mm}^{3}$, CD4 count $<200$ cells $/ \mathrm{mm}^{3}$, CD4 count $200-349$ cells $/ \mathrm{mm}^{3}$, and CD4 count 350-499 cells $/ \mathrm{mm}^{3}$ were respectively associated with increased hazard of hospitalization by 184, 67 and $41 \%$. Somewhat similar to our findings, Crum-Cianflone et al. [13], in an earlier work on this cohort, had found that CD4 count $>499$ cells $/ \mathrm{mm}^{3}$ reduced the hazard of hospitalization when compared to $\mathrm{CD} 4<350$ cells $/ \mathrm{mm}^{3}$ but not 350-499 cells $/ \mathrm{mm}^{3}$. The reason for the difference with our current study may be related to differences in the time frame studied (2006-2010 [current study] versus 1999-2007 [previous study]) or in study design (first 
Table 3 Multivariate Cox Regression Model for Hazard of Hospitalization for Terciles of PCSS and MCSS

\begin{tabular}{|c|c|c|c|c|c|c|}
\hline \multirow[t]{2}{*}{ Variable $^{a}$} & \multicolumn{2}{|c|}{ Model 1: PCSS Model } & \multicolumn{2}{|c|}{ Model 2: MCSS Model } & \multicolumn{2}{|c|}{ Model 3: Combined PCSS and MCSS Model } \\
\hline & $\mathrm{HR}$ & $95 \% \mathrm{Cl}$ & $H R$ & $95 \% \mathrm{Cl}$ & $\mathrm{HR}$ & $95 \% \mathrm{Cl}$ \\
\hline \multicolumn{7}{|l|}{ PCSS } \\
\hline Lower Tercile & 2.18 & $1.64-2.90$ & & & 2.12 & $1.59-2.84$ \\
\hline Middle Tercile & 1.62 & $1.21-2.17$ & & & 1.59 & $1.19-2.14$ \\
\hline Upper Tercile & 1.0 & - & & & 1.0 & - \\
\hline \multicolumn{7}{|l|}{ MCSS } \\
\hline Lower Tercile & & & 1.44 & $1.10-1.87$ & 1.33 & $1.02-1.73$ \\
\hline Middle Tercile & & & 1.14 & $0.87-1.49$ & 1.20 & $0.91-1.57$ \\
\hline Upper Tercile & & & 1.0 & - & 1.0 & - \\
\hline \multicolumn{7}{|l|}{ CD4 Count } \\
\hline$<200$ cells/mm3 & 2.84 & $1.96-4.12$ & 2.99 & $2.06-4.33$ & 2.84 & $1.96-4.12$ \\
\hline 200-349 cells/mm3 & 1.69 & $1.25-2.27$ & 1.67 & $1.24-2.25$ & 1.67 & $1.24-2.26$ \\
\hline 350-499 cells/mm3 & 1.41 & $1.09-1.82$ & 1.37 & $1.05-1.77$ & 1.41 & $1.09-1.83$ \\
\hline > 499 cells/mm3 & 1.0 & - & 1.0 & - & 1.0 & - \\
\hline Plasma Viral Load > 50 Copies/mL & 1.83 & $1.47-2.28$ & 1.88 & $1.51-2.34$ & 1.82 & $1.46-2.26$ \\
\hline Mental Comorbidity & 1.31 & $1.04-1.63$ & 1.30 & $1.04-1.64$ & 1.23 & $0.98-1.55$ \\
\hline AIDS Diagnosis & 1.35 & $1.00-1.83$ & 1.47 & $1.09-1.99$ & 1.34 & $0.99-1.81$ \\
\hline \multicolumn{7}{|l|}{ Rank } \\
\hline Civilian/Retired & 2.06 & $1.26-3.37$ & 2.16 & $1.32-3.52$ & 2.04 & $1.25-3.34$ \\
\hline Enlisted & 1.18 & $0.74-1.88$ & 1.17 & $0.73-1.86$ & 1.19 & $0.74-1.89$ \\
\hline Officer & 1.0 & - & 1.0 & - & - & - \\
\hline Time Since HIV (Per year) & 0.94 & $0.92-0.96$ & 0.94 & $0.93-0.96$ & 0.94 & $0.93-0.96$ \\
\hline
\end{tabular}

${ }^{\mathrm{a}}$ All variables are time-varying

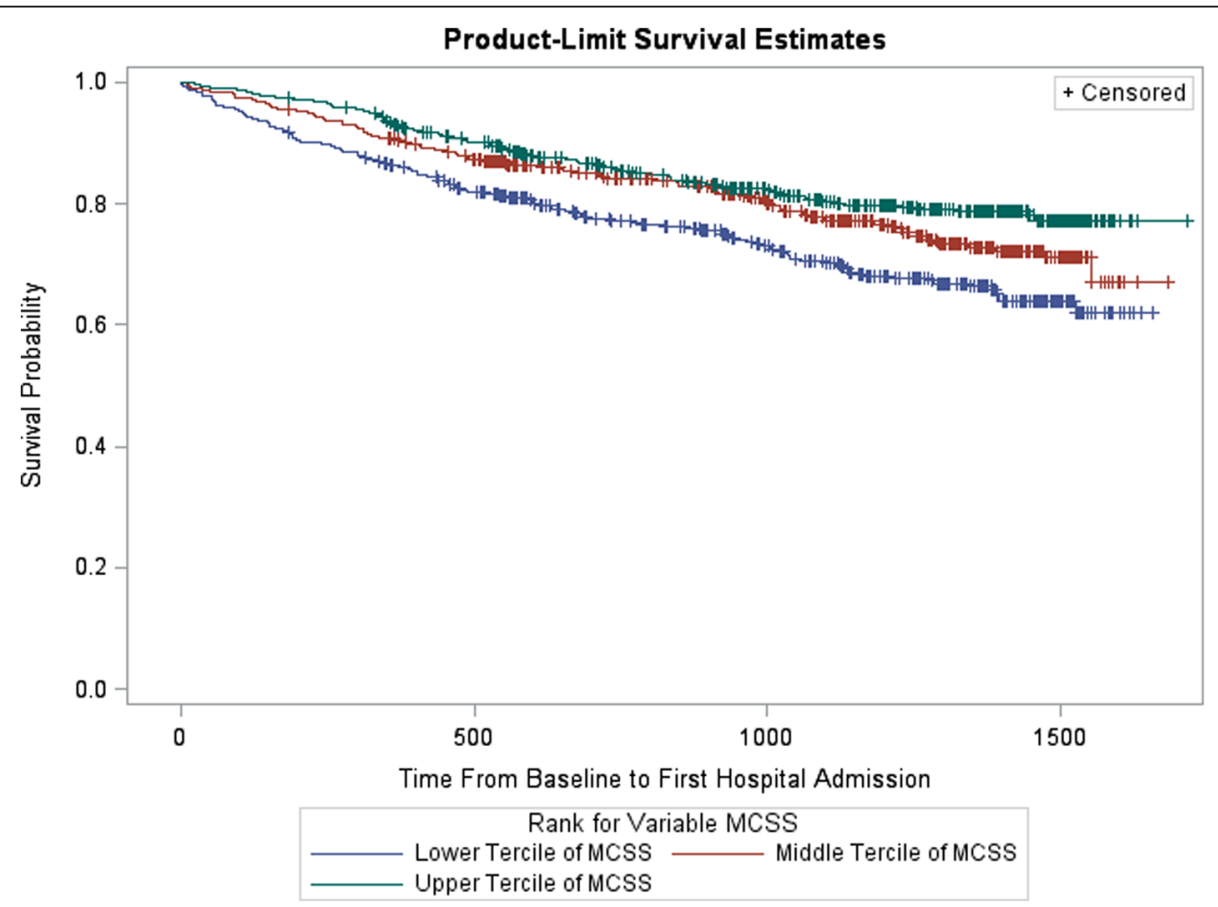

Fig. 2 Kaplan-Meier Survival Curve for Mental Component Summary Score (MCSS). Legend: - Lower Tercile of MCSS, Middle Tercile of MCSS, Upper Tercile of MCSS 
Table 4 Overall Rates of Hospitalization in the NHS and by Terciles of PCSS and MCSS

\begin{tabular}{llllll}
\hline HRQOL & $\begin{array}{l}\text { Terciles } \\
\text { of HRQOL }\end{array}$ & $\begin{array}{l}\text { Total Participants } \\
\text { at Baseline }\end{array}$ & $\begin{array}{l}\text { Total No. Hospitalized } \\
\text { during Follow Up }\end{array}$ & $\begin{array}{l}\text { Duration of } \\
\text { Follow Up (Years) }\end{array}$ & $\begin{array}{l}\text { Rate of Hospitalization } \\
\text { Per 100 Person - Years }\end{array}$ \\
\hline PCSS and MCSS & Overall & 1711 & 366 & 4201.67 & 8.71 \\
PCSS & Lower & 570 & 168 & 1312.34 & 12.80 \\
& Middle & 572 & 124 & 1414.88 & 8.76 \\
& Upper & 569 & 74 & 1474.44 & 5.02 \\
MCSS & Lower & 569 & 152 & 1293.77 & 11.75 \\
& Middle & 571 & 116 & 1397.09 & 8.30 \\
& Upper & 571 & 98 & 1510.8 & 6.49 \\
\hline
\end{tabular}

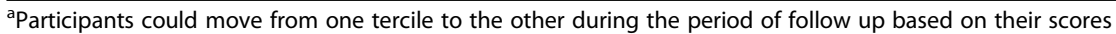

hospitalization [current study] versus all hospitalizations [previous study]) [13]. Other investigators have also shown that lower CD4 count is associated with hospitalization, especially when CD4 count falls below 200 [29-34]. HIV pVL $>50$ copies $/ \mathrm{mL}$ was also associated with hospitalization in our cohort. Although the levels of dichotomization differed, Fielden et al. [34] also found that higher pVL was associated with hospitalization while Mocroft et al. [29] demonstrated that in the last of three time points in their study, there was an increased odds of hospitalization for every log unit increase in pVL.

Interestingly, a longer time since HIV diagnosis was predictive of a reduced hazard of hospitalization. One plausible explanation for this finding may be that individuals with longer disease duration may be more experienced with dealing with symptoms (including subtle ones) associated with their infection and therefore more likely to seek medical attention earlier before admission is warranted. Survival bias may also play a role as those who live longer due to their innate ability to cope with the disease are less likely to be hospitalized because of their relatively healthier state. The finding that being civilian/retired was associated with over $100 \%$ increased hazard of hospitalization is not totally unexpected as this group is older, and age has been associated with higher inpatient admissions [35]. However, the fact that age was not independently associated with hospitalization in our current study may partly reflect the relatively young age of our cohort (median age 42 years; IQR 34 to 49 years) but may also be suggestive of the fact that other factors associated with non-active duty status, such as health related behaviors, played a more important role in this respect. Although being civilian/retired has been shown to be associated with lower PCSS in our cohort [36], its association with hospitalization in the current study is independent of

Table 5 Multivariate Cox Regression Model for Hazard of Hospitalization (PCSS and MCSS Numeric)

\begin{tabular}{|c|c|c|c|c|c|c|}
\hline \multirow[t]{2}{*}{$\overline{\text { Variable }^{a}}$} & \multicolumn{2}{|c|}{ Model 4: PCSS Model } & \multicolumn{2}{|c|}{ Model 5: MCSS Model } & \multicolumn{2}{|c|}{ Model 6: Combined PCSS and MCSS Model } \\
\hline & $\overline{H R}$ & $95 \% \mathrm{Cl}$ & $\mathrm{HR}$ & $95 \% \mathrm{Cl}$ & $\mathrm{HR}$ & $95 \% \mathrm{Cl}$ \\
\hline PCSS, 5 Unit Increments & 0.87 & $0.83-0.92$ & & & 0.88 & $0.84-0.93$ \\
\hline MCSS, 5 Unit Increments & & & 0.91 & $0.87-0.96$ & 0.94 & $0.89-0.99$ \\
\hline \multicolumn{7}{|l|}{ CD4 Count } \\
\hline$<200$ cells/mm3 & 2.76 & $1.90-4.01$ & 2.96 & $2.04-4.29$ & 2.73 & $1.88-3.97$ \\
\hline 200-349 cells/mm3 & 1.66 & $1.23-2.23$ & 1.67 & $1.24-2.25$ & 1.65 & $1.22-2.23$ \\
\hline 350-499 cells/mm3 & 1.38 & $1.07-1.80$ & 1.37 & $1.05-1.77$ & 1.38 & $1.07-1.79$ \\
\hline > 499 cells/mm3 & 1.0 & - & 1.0 & - & 1.0 & - \\
\hline Plasma Viral Load > 50 Copies/mL & 1.86 & $1.49-2.32$ & 1.89 & $1.52-2.36$ & 1.86 & $1.49-2.31$ \\
\hline Mental Comorbidity & 1.31 & $1.05-1.64$ & 1.28 & $1.02-1.61$ & 1.23 & $0.97-1.54$ \\
\hline AIDS Diagnosis & 1.34 & $0.99-1.82$ & 1.48 & $1.09-2.00$ & 1.34 & $0.99-1.82$ \\
\hline \multicolumn{7}{|l|}{ Rank } \\
\hline Civilian/Retired & 2.21 & $1.35-3.61$ & 2.14 & $1.31-3.49$ & 2.15 & $1.32-3.52$ \\
\hline Enlisted & 1.28 & $0.80-2.04$ & 1.16 & $0.73-1.85$ & 1.27 & $0.79-2.02$ \\
\hline Officer & 1.0 & - & 1.0 & - & - & - \\
\hline Time Since HIV (Per year) & 0.94 & $0.92-0.96$ & 0.95 & $0.93-0.96$ & 0.94 & $0.93-0.96$ \\
\hline
\end{tabular}

${ }^{\mathrm{a}}$ All variables are time-varying 
PCSS (Tables 3 and 5). Finally, while HAART was significant in the unadjusted model, it was no longer significant after adjustment showing that its effect may have been captured by other variables.

Potential limitations of our study include the predominantly male patients in the NHS cohort which may limit its generalizability to females. The regimented lifestyle of our military population and the requirements of physical and mental fitness may also mean that they may promptly seek medical attention, a behavior that may not necessarily be seen in the general population. We may not have captured hospital admissions outside the military settings; however, with the use of trained coordinators to conduct participants' interviews, the number of missed admissions outside the single payer electronic health records systems of the U.S. military is expected to be small. Because we administratively censored participants after September 2010, follow-up was limited for those subjects surveyed in 2010; however, as subjects were censored 6 months after their last survey, this affected only those enrolled in $2010(n=99)$ [36]. In sensitivity analysis we excluded these 99 participants and our results remained essentially the same. In this current study, we did not specifically evaluate whether within-person difference in HRQOL score may predict hospitalization. While such difference in score may be more meaningful to the clinician interested in individual risk of inpatient admission over time, we believe that knowing the actual scores that predict hospitalization is a useful starting point. More so, within-person difference in score may be dependent on the baseline HRQOL score. Finally, our findings that PCSS and MCSS were predictive of hospitalization should not be interpreted as implying causation but instead that physical and mental functional health may be surrogates for the actual causes of hospitalization. Yet, the fact that these measures were statistically significant in predicting hospitalization in all multivariate models, the magnitude of the effect sizes, and the dose response relationships support their utility in clinical practice.

Our study had major advantages. There was clearly an established temporal relationship between the HRQOL scores and hospitalization. Because hospitalization may also negatively impact HRQOL [37], we further conducted sensitivity analyses to rule out reverse causation by excluding participants whose scores were taken within 1 week hospitalization but our findings remained essentially the same. This temporal pattern was also true for the other time-dependent covariates included in the study. The clear dose-response relationship between PCSS and hospitalization in our study strongly supports its utility as a predictive tool. To further demonstrate the discriminatory quality of PCSS we used it as a continuous variable, and it showed that for every 5-unit increase in PCSS the hazard of hospitalization was reduced by $12 \%$ (Table 5). MCSS, on the other hand, reduced the hazard of hospitalization by $6 \%$ for every 5 -unit increase in MCSS. Our study therefore adds to the predictive utility of the HRQOL measures in HIV-infected persons as assessed by the SF-36. Other important strengths of our study include its large sample size, open access to healthcare and medications, racial diversity, and the heterogeneity of the cohort with regards to the range of values for HIV disease indicators (CD4 count and pVL), and other clinical parameters, such as medical and mental comorbidities with respective prevalence of 17 and $29 \%$ of the cohort at baseline. The heterogeneity of the cohort (Table 1) is further reflected by the wide range of PCSS (16.7 to 70.7) and MCSS (8.8 to 67.8), a finding that is rare in other predictive studies using the SF-36 that tend to be with persons with relatively very low scores $[3,4,6,28,38]$. Finally, HRQOL summary scores may be useful in prognostic studies in HIV-infected populations because they capture information beyond HIV disease-specific indicators, such as CD4 count and pVL. The causes of hospitalization in HIV-infected individuals are now beyond disease-related factors but include both medical and surgical conditions, especially in the HAART era [13]. HRQOL is also reflective of perceptions that may potentially affect subsequent health-seeking behaviors and utilization of healthcare resources including preventive services $[4,39]$.

\section{Conclusion}

After controlling for factors associated with hospitalization among those with HIV, both PCSS and MCSS were predictive of all-cause hospitalization in the NHS cohort with similar effect sizes for PCSS and low CD4. Applying innovative strategies to improve modifiable risk factors that influence the physical and mental functional status of HIV-infected individuals has the potential to reduce the rate of hospitalization in this patient population. We, therefore, recommend the use of HRQOL in stratifying hospitalization risk among HIV-infected individuals. Finally, future research to evaluate whether within-person difference in HRQOL scores is predictive of hospitalization is needed, as this may even increase the utility of HRQOL measures in clinical settings.

\section{Abbreviations \\ HAART: Highly Active Antiretroviral Therapy; HIV: Human Immunodeficiency Virus; HRQOL: Health-Related Quality of Life; MCSS: Mental Component Summary Scores; NPI-HAART: Non-Protease Inhibitor HAART; PCSS: Physical Component Summary Scores; PI-HAART: Protease Inhibitor HAART; pVL: Plasma Viral Load; SF-36: Short Form 36}

\section{Acknowledgements}

Support for this work (IDCRP-000-24) was provided by the Infectious Disease Clinical Research Program (IDCRP), a Department of Defense (DoD) program executed through the Uniformed Services University of the Health Sciences. 
This project has been funded in whole, or in part, with federal funds from the National Institute of Allergy and Infectious Diseases, National Institutes of Health (NIH), under Inter-Agency Agreement Y1-Al-5072.

Members of the Infectious Disease Clinical Research Program HIV Working Group include the following:

Madigan Army Medical Center, Tacoma, WA: S. Chambers; COL (Ret) M.

Fairchok; LTC A. Kunz; C. Schofield.

National Institute of Allergy and Infectious Diseases, Bethesda, MD: J. Powers; COL (Ret) E. Tramont.

Naval Medical Center, Portsmouth, VA: S. Banks; CDR K. Kronmann; T. Lalani; LCDR K. St. Clair; R. Tant.

Naval Medical Center, San Diego, CA: CAPT M. Bavaro; R. Deiss; A. Diem; N. Kirkland; CDR R. Maves.

San Antonio Military Medical Center, San Antonio, TX: S. Merritt; T. O'Bryan; LtCol J. Okulicz; C. Rhodes; J. Wessely.

Tripler Army Medical Center, Honolulu, HI: COL T. Ferguson; LTC J. HawleyMolloy.

Uniformed Services University of the Health Sciences, Bethesda, MD: B. Agan; M. Byrne; X. Chu; M. Glancey; G. Macalino; E. Parmelee; COL (Ret) J. Pavlin; X. Wang; S. Won; P. Wright.

Walter Reed Army Institute of Research, Silver Spring, MD: S. Peel. Walter Reed National Military Medical Center, Bethesda, MD: MAJ J. Blaylock; H. Burris; C. Decker; A. Ganesan; LTC R. Ressner; D. Wallace; CAPT T. Whitman. The authors would also like to thank Dr. John W. Harrington for reading the initial manuscript and making valuable suggestions.

\section{Funding}

This project has been funded in whole, or in part, with federal funds from the National Institute of Allergy and Infectious Diseases, National Institutes of Health (NIH), under Inter-Agency Agreement Y1-Al-5072.

\section{Availability of data and materials}

The authors confirm that all data underlying the findings are fully available without restriction. Data for this study is located at and can be requested from Infectious Disease Clinical Research Program (IDCRP), headquartered at the Uniformed Services University (USU), Department of Preventive Medicine and Biostatistics. Address: 11300 Rockville Pike, Suite 600, Rockville, MD 20852; Email: idcrp@idcrp.org.

\section{Authors' contributions}

Conceptualization: LE SW GM BKA. Data curation: BKA GM IDCRPHIVWG Formal analysis: LE. Investigation: LE SW MP AAE GM BKA IDCRPHIVWG. Methodology: LE SW MP AAE GM BKA. Project administration: LE SW BKA IDCRPHIVWG. Resources: IDCRPHIVWG. Software: LE IDCRPHIIWG. Supervision: SW MP AAE BKA. Validation: LE SW MP AAE GM BKA. Visualization: LE. Writing - original draft: LE SW BKA. Writing - review \& editing: LE SW MP AAE GM BKA IDCRPHIVWG. All authors contributed to the content of the manuscript and concurred with the decision to submit it for publication.

\section{Ethics approval and consent to participate}

The U.S. Military HIV Natural History Study has been approved centrally by the Uniformed Services University Institutional Review Board (IRB) and at each participating site and is conducted according to the principles expressed in the Declaration of Helsinki. Written informed consent was obtained from the participants. This analysis was approved by the central IRB and Drexel University.

\section{Competing interests}

The authors declare that they have no competing interests.

\section{Publisher's Note}

Springer Nature remains neutral with regard to jurisdictional claims in published maps and institutional affiliations.

\section{Author details}

'Department of Epidemiology and Biostatistics, Dornsife School of Public Health, Drexel University, Philadelphia, PA, USA. ${ }^{2}$ Department of Pediatrics, Eastern Virginia Medical School, Norfolk, VA, USA. ${ }^{3}$ Children's Hospital of the King's Daughters, 601 Children's Lane, Norfolk, VA 23507, USA. ${ }^{4}$ Infectious Disease Clinical Research Program, Department of Preventive Medicine and
Biostatistics, Uniformed Services University of the Health Sciences, Bethesda, MD, USA. ${ }^{5}$ Henry M. Jackson Foundation for the Advancement of Military Medicine, Inc., Bethesda, MD, USA.

Received: 25 October 2017 Accepted: 10 May 2018

Published online: 30 May 2018

\section{References}

1. Gakhar H, Kamali A, Holodniy M. Health-related quality of life assessment after antiretroviral therapy: a review of the literature. Drugs. 2013;73(7):651-72.

2. Degroote $S$, Vogelaers $D$, Vandijck DM. What determines health-related quality of life among people living with HIV: an updated review of the literature. Arch Public Health. 2014;72(1):40.

3. Cunningham WE, Crystal S, Bozzette S, Hays RD. The association of healthrelated quality of life with survival among persons with HIV infection in the United States. J Gen Intern Med. 2005;20(1):21-7.

4. de Boer-van der Kolk IM, Sprangers MA, Prins JM, Smit C, de Wolf F, Nieuwkerk PT. Health-related quality of life and survival among HIV-infected patients receiving highly active antiretroviral therapy: a study of patients in the AIDS therapy evaluation in the Netherlands (ATHENA) cohort. Clin Infect Dis. 2010;50(2):255-63.

5. Mathews WC, May S. EuroQol (EQ-5D) measure of quality of life predicts mortality, emergency department utilization, and hospital discharge rates in HIV-infected adults under care. Health Qual Life Outcomes. 2007;5:5.

6. Jacobson DL, Wu AW, Feinberg J. Health-related quality of life predicts survival, cytomegalovirus disease, and study retention in clinical trial participants with advanced HIV disease. J Clin Epidemiol. 2003;56(9):874-9.

7. Mocroft A, Vella S, Benfield TL, et al. Changing patterns of mortality across Europe in patients infected with HIV-1. EuroSIDA Study Group. Lancet. 1998:352(9142):1725-30

8. Palella FJ Jr, Delaney KM, Moorman AC, et al. Declining morbidity and mortality among patients with advanced human immunodeficiency virus infection. HIV outpatient study investigators. N Engl J Med. 1998;338(13):853-60.

9. Centers for Disease Control and Prevention (CDC). Rates of diagnoses of HIV infection among adults and adolescents, by area of residence, 2011_United States and 6 dependent areas. 2011; https://www.cdc.gov/hiv/pdf/statistics_ 2011_HIV_Surveillance_Report_vol_23.pdf. Accessed 26 Jan 2015.

10. Singh JA, Borowsky SJ, Nugent S, et al. Health-related quality of life, functional impairment, and healthcare utilization by veterans: veterans' quality of life study. J Am Geriatr Soc. 2005;53(1):108-13.

11. Singh JA, Nelson DB, Fink HA, Nichol KL. Health-related quality of life predicts future health care utilization and mortality in veterans with selfreported physician-diagnosed arthritis: the veterans arthritis quality of life study. Semin Arthritis Rheum. 2005;34(5):755-65.

12. Centers for Disease Control and Prevention. Measuring Healthy Days. Atlanta: CDC; 2000.

13. Crum-Cianflone NF, Grandits G, Echols S, et al. Trends and causes of hospitalizations among HIV-infected persons during the late HAART era: what is the impact of CD4 counts and HAART use? J Acquir Immune Defic Syndr. 2010;54(3):248-57.

14. Chun HM, Fieberg AM, Hullsiek KH, et al. Epidemiology of hepatitis B virus infection in a US cohort of HIV-infected individuals during the past 20 years. Clin Infect Dis. 2010;50(3):426-36.

15. Chun HM, Roediger MP, Hullsiek KH, et al. Hepatitis B virus coinfection negatively impacts HIV outcomes in HIV seroconverters. J Infect Dis. 2012;205(2):185-93.

16. Pelak K, Goldstein DB, Walley NM, et al. Host determinants of HIV-1 control in African Americans. J Infect Dis. 2010;201(8):1141-9.

17. Spaulding AB, Lifson AR, Iverson ER, et al. Gonorrhoea or chlamydia in a U.S. military HIV-positive cohort. Sex Transm Infect. 2012;88(4):266-71.

18. RAND. Scoring instructions for the 36-item short form survey (SF-36). https://www.rand.org/health/surveys_tools/mos/36-item-short-form/scoring. html. Accessed 05/5/2013.

19. Hays Ron D, Sherbourne CD, Spritzer K, Dixon WJ. A Microcomputer Program (sf36.exe) that Generates SAS Code for Scoring the SF-36 Health Survey. Santa Monica, CA: RAND Corporation; 1996. https://www.rand.org/ pubs/drafts/DRU1437.html.

20. Potard V, Chassany O, Lavignon M, Costagliola D, Spire B. Better health-related quality of life after switching from a virologically effective regimen to a regimen containing efavirenz or nevirapine. AIDS Care. 2010;22(1):54-61. 
21. Fumaz CR, Tuldra A, Ferrer MJ, et al. Quality of life, emotional status, and adherence of HIV-1-infected patients treated with efavirenz versus protease inhibitor-containing regimens. J Acquir Immune Defic Syndr. 2002;29(3):244-53.

22. Campo RE, Cohen C, Grimm K, Shangguan T, Maa J, Seekins D. Switch from protease inhibitor- to efavirenz-based antiretroviral therapy improves quality of life, treatment satisfaction and adherence with low rates of virological failure in virologically suppressed patients. Int J STD AIDS. 2010;21(3):166-71.

23. Cox D.R. Regression Models and Life Tables. J Royal Statistical Society. 1972;Series B34(2):34

24. Allison PD. Estimating Cox Regression Models with PROC PHREG. Survival Analysis Using SAS: A Parictical Guide. Cary, NC: SAS Institute Inc.; 2010. p. 125-201.

25. Bellera CA, MacGrogan G, Debled M, de Lara CT, Brouste V, MathoulinPelissier S. Variables with time-varying effects and the cox model: some statistical concepts illustrated with a prognostic factor study in breast cancer. BMC Med Res Methodol. 2010;10:20.

26. Mapes DL, Bragg-Gresham JL, Bommer J, et al. Health-related quality of life in the Dialysis outcomes and practice patterns study (DOPPS). Am J Kidney Dis. 2004;44(5 Suppl 2):54-60.

27. Lowrie EG, Curtin RB, LePain N, Schatell D. Medical outcomes study short form-36: a consistent and powerful predictor of morbidity and mortality in dialysis patients. Am J Kidney Dis. 2003;41 (6):1286-92.

28. Schron E, Friedmann E, Thomas SA. Does health-related quality of life predict hospitalization or mortality in patients with atrial fibrillation? J Cardiovasc Electrophysiol. 2014;25(1):23-8.

29. Mocroft A, Monforte A, Kirk O, et al. Changes in hospital admissions across Europe: 1995-2003. Results from the EuroSIDA study. HIV medicine. 2004; 5(6):437-47.

30. Paul S, Gilbert HM, Lande L, et al. Impact of antiretroviral therapy on decreasing hospitalization rates of HIV-infected patients in 2001. AIDS Res Hum Retrovir. 2002;18(7):501-6.

31. Buchacz K, Baker RK, Moorman AC, et al. Rates of hospitalizations and associated diagnoses in a large multisite cohort of HIV patients in the United States, 1994-2005. AIDS. 2008;22(11):1345-54.

32. Krentz HB, Dean S, Gill MJ. Longitudinal assessment (1995-2003) of hospitalizations of HIV-infected patients within a geographical population in Canada. HIV medicine. 2006:7(7):457-66.

33. Gebo KA, Fleishman JA, Moore RD. Hospitalizations for metabolic conditions, opportunistic infections, and injection drug use among HIV patients: trends between 1996 and 2000 in 12 states. J Acquir Immune Defic Syndr. 2005;40(5):609-16.

34. Fielden SJ, Rusch ML, Levy AR, et al. Predicting hospitalization among HIVinfected antiretroviral naive patients starting HAART: determining clinical markers and exploring social pathways. AIDS Care. 2008:20(3):297-303.

35. National Center for Health Statistics. Health, United States, 2015: with special feature on racial and ethnic health disparities. 2016.

36. Emuren $L$, Welles $S$, Evans AA, et al. Health-related quality of life among military HIV patients on antiretroviral therapy. PLoS One. 2017;12(6): e0178953.

37. Globe DR, Hays RD, Cunningham WE. Associations of clinical parameters with health-related quality of life in hospitalized persons with HIV disease. AIDS Care. 1999;11(1):71-86.

38. Mapes DL, Lopes AA, Satayathum S, et al. Health-related quality of life as a predictor of mortality and hospitalization: the Dialysis outcomes and practice patterns study (DOPPS). Kidney Int. 2003;64(1):339-49.

39. Idler EL, Kasl SV. Self-ratings of health: do they also predict change in functional ability? J Gerontol Ser B Psychol Sci Soc Sci. 1995;50((6):S344-53.

\section{Ready to submit your research? Choose BMC and benefit from:}

- fast, convenient online submission

- thorough peer review by experienced researchers in your field

- rapid publication on acceptance

- support for research data, including large and complex data types

- gold Open Access which fosters wider collaboration and increased citations

- maximum visibility for your research: over $100 \mathrm{M}$ website views per year

At BMC, research is always in progress.

Learn more biomedcentral.com/submissions 